\title{
ESTIMATION OF COSTS AND RETURNFOR THE ORGANIC GARLIC IN NEWLY CULTIVATED SOIL
}

\author{
ABDEL-RASHEED, K. G. ${ }^{2}$, Y. M. M. MOUSTAFA ${ }^{1}$, E. A. HASSAN ${ }^{2}$, \\ Y. Y. ABDEL-ATI ${ }^{1}$ and S. G. H. GADEL-HAK ${ }^{1}$
}

1. Horticulture Department, Faculty of Agriculture, Minia University, Minia, Egypt.

2. Central Laboratory of Organic Agriculture (CLOA), ARC, Giza, Egypt.

(Manuscript received 24 August 2016)

\begin{abstract}
A preliminary study focuses on economic analysis of organic garlic production in new cultivated soil under El-Minia Governorate conditions. Data were collected from growing 21 garlic genotypes under four compost levels. The obtained results showed that the total estimated cost of production for one feddan $\left(4200 \mathrm{~m}^{2}\right)$ of organic garlic was ranged from 9382.25 to 12641.5 L.E for fresh yield and from 9882.25 to 13141.5 L.E for cured yield. In this study, the total gross for the production of fresh yield ranged from 0.9803 to 5.036 ton/feddan. For cured yield, it ranged from 0.633 to 3.445 ton/feddan. In conclusion, colored cultivar "Egaseed 1" or "El-Wady" white clone were the highest profitable genotypes under the condition of the present study.
\end{abstract}

Keywords: Allium sativum L., Genotypes, Compost, Organic production, Economics.

\section{INTRODUCTION}

Several academic institutions, researchers, nonprofit agencies and farmers have created budgets on a variety of vegetable crops. The University of California at Davis (2008) has perhaps the greatest number of such studies but these may be of limited value to growers outside of California, USA. However, an understanding of production costs is critical for decision makers. These types of analyses help farm managers understand and identify profitable enterprises (Frank, 1997; Toaima et al., 2001; Besheit et al., 2002; Estes et al., 2003; Conner and Rangarajan, 2009; Dahshan, 2013 and Abd El-Kareem, 2015). They concluded that many factors, such as crop rotation, soil type, marketing and production costs are vital information for production and pricing decisions.

The critical part of crop budget was the calculation of costs related to labor, field management, pesticides and fertilization. Pepper production was found to be a profitable enterprise considering the profit realized by farmers in the study area as reported by Sanusi and Ayinde (2013). 
Hasan et al. (2012) estimated the benefit cost ratio for garlic (1.85) and showed that garlic cultivation was profitable. However, garlic cultivation requires a high level of working capital and human labor (Meena et al., 2013).

Growth in organic food sales was 53\% in the United states between 2005 and 2008 (Richards, 2011). Increased demand is motivating worldwide. More benefits can be gained from organic agriculture. These benefits due to a reduction of the use of synthetic chemical and fertilizers, which can reduce toxic chemical exposure and possibly reduce input costs. Also, the possible price premiums and growing value added markets are very important elements in the stability of organic products (Post and Schahczenski, 2012). Thus, improving the quality and quantity of organic products production and increased yield productivity are very important elements. Cost information will help the organic farmers to face marketing risks of organic garlic. The present investigation was carried out to estimate the organic garlic production cost and return under cultivated the new soil conditions to attract more organic garlic investors.

\section{MATERIALS AND METHODS}

The present study was carried out during the three successive winter seasons of 2011/2012/, 2012/2013 and 2013/2014 at the Experimental Farm of Central Laboratory of Organic Agriculture (CLOA) at Village 8, El- Minia, Egypt in new reclaimed soil.

The rate of application was based on $\mathrm{N}$ equivalent dose (120 and $60 \mathrm{~kg}$ total $\mathrm{N}$ per feddan).

The experimental design was split plot design with three replications. Commercial plant compost "El-Nil" and Plant- animal compost "El-Obour" each at two rates were distributed in the four main plots.

Compost El-Nil was applied at the rate of 10.666 and 5.333 tons/fed in the first season and 9.740 and 4.870 tons per feddan in the second one whereas compost El-Obour at the rate of 13.698 and 6.849 tons per feddan in the first season and 16.666 and 8.333 tons/feddan in the second season, respectively. Samples of ripe plant compost and plant+animal compost were subjected to chemical analysis and the results are presented in Table (1). 
Table 1. The physical and chemical properties of the organic manure

\begin{tabular}{|l|c|c|c|c|}
\hline \multirow{2}{*}{ Parameter } & \multicolumn{2}{|c|}{ El-Nil compost } & \multicolumn{2}{c|}{ El- Obour compost } \\
\cline { 2 - 5 } & First season & Second season & First season & Second season \\
\hline Weight of m3 dry, kg & 600 & 620 & 700 & 700 \\
\hline Moisture, \% & 25 & 23 & 27 & 28 \\
\hline Organic matter, \% & 30 & 32 & 28 & 27 \\
\hline Organic Carbon, \% & 23 & 25 & 18 & 18 \\
\hline $\mathrm{pH}$ & 7.1 & 7.2 & 7.4 & 7.5 \\
\hline EC (ds/m) & 3 & 3.4 & 3.5 & 3.5 \\
\hline C/N ratio & $1: 15.5$ & $1: 16$ & $1: 17$ & $1: 17$ \\
\hline Total N, \% & 1.5 & 1.6 & 1.2 & 1 \\
\hline Total P, \% & 0.8 & 0.85 & 0.65 & 0.6 \\
\hline Total K, \% & 1 & 1 & 0.85 & 0.80 \\
\hline Total Fe (ppm) & $1500-2000$ & $1500-2000$ & $1000-1800$ & $1000-1800$ \\
\hline Total Mn (ppm) & $100-150$ & $100-150$ & $80-120$ & $80-120$ \\
\hline Total Cu (ppm) & $160-240$ & $160-240$ & $100-160$ & $100-160$ \\
\hline Total Zn $(\mathrm{ppm})$ & $40-80$ & $40-80$ & $30-50$ & $30-50$ \\
\hline Weed seed & Nothing & Nothing & Nothing & Nothing \\
\hline Nematodes & Nothing & Nothing & Nothing & Nothing \\
\hline Parasites & Nothing & Nothing & Nothing & Nothing \\
\hline
\end{tabular}

The 21 garlic genotypes were randomly arranged in the sub-plots. The sources and colors of the tested garlic genotypes were listed in (Table 2). No synthetic fertilizers and pesticides were used.

Uncultivated soil received the estimated amount of compost after ploughing and harrowing to pulverize the soil before making the ridges units. Main plots occupied the four compost treatments and garlic genotypes were randomly distributed in the subplots. Each main plot had12 rows and each plot area was $7.20 \mathrm{~m} \times 33 \mathrm{~m}$. Spacing was $10 \mathrm{~cm}$ between cloves within the row and $60 \mathrm{~cm}$ between rows. Garlic cloves were hand planted on both sides of each ridge on 10th and 15th of October 2012 and 2013, respectively. However the seed-cloves of all genotypes were produced organically in the previous season using plant compost equivalent to $120 \mathrm{~kg}$ total N/feddan as the only source of nutrients in 2011/2012.

In each replicate, the studied treatments were the product of 4 compost levels $\times 21$ garlic genotypes $=84$ treatments. Each treatment was replicated three times and planted in two rows, and each row contained 60 cloves.

Cost of organic garlic production was estimated and compared among the 84 treatments which are the combinations among 21 garlic genotypes and four levels of compost. These treatments were distributed in split-plot design with three replications. All input costs were taken into consideration for computing the production cost which include: compost (plant compost and plant-animal compost), seed, soil preparations, planting, hoeing, irrigation, harvesting and rent values. The local and exportable garlic prices per kilogram were considered to be 3.5-5.6 L.E for 
white and 4.5-6.6 L.E for coloured type (Dr. Osman, Dr. Abdel-Moity and Mr. Hegazy Personal Communication). Economic analyses were performed to estimate returns and profitability using the following formula according to O'Dell (1984) and Hanque et al. (2013) with some modification:

$*$ Gross $=$ Total yield $x$ Price

$*$ Net return $(\mathrm{NR})=$ Gross - Total cost

* Profitability, \%= (NR/TC)*100

Table 2. Source, skin colour and bulb type of the tested garlic genotypes

\begin{tabular}{|c|c|}
\hline Coloured genotypes & Source \\
\hline Aiat clone $1 *$ & $\begin{array}{l}\text { Sids Horticultural Research Station, Agricultural Research Center, Giza, } \\
\text { Egypt. }\end{array}$ \\
\hline BaniGhany & $\begin{array}{l}\text { Sids Horticultural Research Station, Agricultural Research } \\
\text { Center, Giza, Egypt. }\end{array}$ \\
\hline Clone 21 & The Agricultural Egyptian Company for Seed Production, Egypt. \\
\hline Clone 22 & The Agricultural Egyptian Company for Seed Production, Egypt. \\
\hline Egaseed 1 & The Agricultural Egyptian Company for Seed Production, Egypt. \\
\hline Egaseed 2 & The Agricultural Egyptian Company for Seed Production, Egypt. \\
\hline Grower's Clone & $\begin{array}{l}\text { Department of Horticulture, Faculty of Agriculture, Minia University, Minia, } \\
\text { Egypt. }\end{array}$ \\
\hline Salaqus-3 & Sids Horticultural Research Station, Agricultural Research Center, Giza, Egypt. \\
\hline Sids 40 Aiat & Sids Horticultural Research Station, Agricultural Research Center, Giza, Egypt. \\
\hline Sids 40 Station & Sids Horticultural Research Station, Agricultural Research Center, Giza, Egypt. \\
\hline White genotypes & Source \\
\hline Clone 5 & Sids Horticultural Research Station, Agricultural Research Center, Giza, Egypt. \\
\hline Clone 10 & Sids Horticultural Research Station, Agricultural Research Center, Giza, Egypt. \\
\hline Clone 18 & Sids Horticultural Research Station, Agricultural Research Center, Giza, Egypt. \\
\hline Clone 22 & Sids Horticultural Research Station, Agricultural Research Center, Giza, Egypt. \\
\hline Clone 24 & The Agricultural Egyptian Company for Seed Production, Egypt. \\
\hline Clone 25 & The Agricultural Egyptian Company for Seed Production, Egypt. \\
\hline Egaseed clone & The Agricultural Egyptian Company for Seed Production, Egypt. \\
\hline Egyptian & $\begin{array}{l}\text { Department of Horticulture, Faculty of Agriculture, Minia University, Minia, } \\
\text { Egypt. }\end{array}$ \\
\hline Elwady & Sids Horticultural Research Station, Agricultural Research Center, Giza, Egypt. \\
\hline Owainat & Sids Horticultural Research Station, Agricultural Research Center, Giza, Egypt. \\
\hline Salaqus-3 & Sids Horticultural Research Station, Agricultural Research Center, Giza, Egypt. \\
\hline
\end{tabular}

*All genotypes are soft-neck except Egaseed 2 (hard-neck) and Clone 24 (semi hard-neck) 


\section{RESULTS AND DISCUSSION}

\section{Estimation of cost and return for organic garlic production in the study area}

The estimated costs and returns for the production of garlic are shown in Tables (3, 4and 5) and also in Figures 1 and 2. Production costs involved costs of field operations, labor, materials, harvesting and packing as well as land rent. Based on the seed-cloves price for each genotype, the total estimated cost of production per one feddan of organic garlic was ranged from 9382.25 to 12641.5 L.E for fresh yield and from 9882.25 to 13141.5 L.E for cured yield. Seed clovescost are vary among the tested genotypes. However, the price per kilogram of white garlic type was lower than that of coloured one. The cost of plant-animal compost comprised the highest input category followed by labour, land rent and seed cost in descending order. Tables (4 and 5) showed the total gross for the production of fresh yield of organic garlic ranged from 4411.5 L.E for Aiat clone 1 when received $60 \mathrm{~kg}$ total N rate from plant-animal compost to 21082.5 L.E for harvesting cultivar Egaseed 1 from plots received $120 \mathrm{~kg}$ total $\mathrm{N} /$ Feddan level.

The highest net return for the interaction treatments was obtained by Egaseed 1 with $120 \mathrm{~kg}$ total N/Feddan from plant-animal source. Twelve out of 42 plant compost treatments and 13 out of 42 plant-animal compost treatments had negative sign for the net return parameter. These results suggested that there is a need to a better understand of the role of choosing the adaptable cultivar for the type of production. Although, the organic garlic grower incurred a total cost of 12141.5 L.E the grower could earn a profitability rate of $73.64 \%$ (Table 4).

Regarding the cured yield, the estimated gross, net return and profitability parameters are shown in (Table 5). Interesting results were obtained with the assumption proposed that cured yield will be transferred to foreign markets. However, 11 genotypes had negative values under $60 \mathrm{~kg}$ total N/Feddan from plantanimal compost, while only 6ones had negative sign at $120 \mathrm{~kg}$ total $\mathrm{N} /$ Feddan from the same source. On the other side, 9 and 6 genotypes had negative sign at 120 and $60 \mathrm{~kg}$ total N/Feddan from plant compost, respectively.

In conclusion, this study showed that organic garlic production is a profitable enterprise when the coloured cultivar "Egaseed 1" or "El-Wady" white clone are used and fertilized with $120 \mathrm{~kg}$ total N/Feddan from either type of composts and further study should be done to maximize the yield per unit area through using better agricultural practices. In general improving the yield quality and quantity will lowered the economic and marketing risks of organic garlic production and will help to extend the organic garlic growing areas. 
Table 3. Total cost for fresh yield of all garlic genotypes fertilized with different compost treatments.

\begin{tabular}{|c|c|c|c|c|c|c|c|c|c|c|c|c|c|c|c|}
\hline \multirow{3}{*}{ Genotypes } & \multicolumn{4}{|c|}{ Compost cost } & \multirow{3}{*}{ 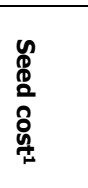 } & \multirow{3}{*}{ 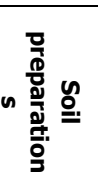 } & \multirow{3}{*}{ 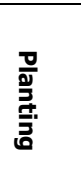 } & \multirow{3}{*}{ 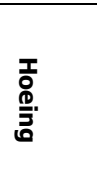 } & \multirow{3}{*}{ 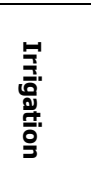 } & \multirow{3}{*}{ 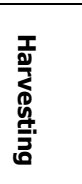 } & \multirow{3}{*}{ 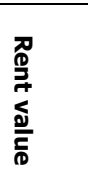 } & \multicolumn{4}{|c|}{ Total cost (TC) L.E/Fed. } \\
\hline & Plant com & ost & $\begin{array}{l}\text { Plant-anin } \\
\text { compost }\end{array}$ & & & & & & & & & \multicolumn{2}{|c|}{ Plant compost } & \multicolumn{2}{|c|}{$\begin{array}{l}\text { Plant-animal } \\
\text { compost }\end{array}$} \\
\hline & $\begin{array}{l}120 \mathrm{~kg} \\
\text { N/Fed }\end{array}$ & $\begin{array}{l}60 \mathrm{~kg} \\
\mathrm{~N} / \mathrm{Fed}\end{array}$ & $\begin{array}{l}120 \text { kg } \\
\text { N/Fed }\end{array}$ & $\begin{array}{l}60 \mathrm{~kg} \\
\mathrm{~N} / \mathrm{Fed}\end{array}$ & & & & & & & & $\begin{array}{l}120 \mathrm{~kg} \\
\text { N/Fed }\end{array}$ & $\begin{array}{l}60 \mathrm{~kg} \\
\mathrm{~N} / \mathrm{Fed}\end{array}$ & $\begin{array}{l}120 \mathrm{~kg} \\
\mathrm{~N} / \mathrm{Fed}\end{array}$ & $\begin{array}{l}60 \mathrm{~kg} \\
\mathrm{~N} / \mathrm{Fed}\end{array}$ \\
\hline Aiat" clone 1 " & 3162.5 & 1581.25 & 3215.5 & 1607.75 & 2250 & 435 & 900 & 2250 & 675 & 750 & 1666 & 12088.5 & 10507.25 & 12141.5 & 10533.75 \\
\hline Bani Ghany & 3162.5 & 1581.25 & 3215.5 & 1607.75 & 2250 & 435 & 900 & 2250 & 675 & 750 & 1666 & 12088.5 & 10507.25 & 12141.5 & 10533.75 \\
\hline Clone 21 & 3162.5 & 1581.25 & 3215.5 & 1607.75 & 2250 & 435 & 900 & 2250 & 675 & 750 & 1666 & 12088.5 & 10507.25 & 12141.5 & 10533.75 \\
\hline Clone 22 C & 3162.5 & 1581.25 & 3215.5 & 1607.75 & 2250 & 435 & 900 & 2250 & 675 & 750 & 1666 & 12088.5 & 10507.25 & 12141.5 & 10533.75 \\
\hline Egaseed 1 & 3162.5 & 1581.25 & 3215.5 & 1607.75 & 2250 & 435 & 900 & 2250 & 675 & 750 & 1666 & 12088.5 & 10507.25 & 12141.5 & 10533.75 \\
\hline Egaseed 2 & 3162.5 & 1581.25 & 3215.5 & 1607.75 & 2750 & 435 & 900 & 2250 & 675 & 750 & 1666 & 12588.5 & 11007.25 & 12641.5 & 11033.75 \\
\hline Grower's Clone & 3162.5 & 1581.25 & 3215.5 & 1607.75 & 2250 & 435 & 900 & 2250 & 675 & 750 & 1666 & 12088.5 & 10507.25 & 12141.5 & 10533.75 \\
\hline Salaqus-3 C & 3162.5 & 1581.25 & 3215.5 & 1607.75 & 2250 & 435 & 900 & 2250 & 675 & 750 & 1666 & 12088.5 & 10507.25 & 12141.5 & 10533.75 \\
\hline Sids 40 "Aiat" & 3162.5 & 1581.25 & 3215.5 & 1607.75 & 2250 & 435 & 900 & 2250 & 675 & 750 & 1666 & 12088.5 & 10507.25 & 12141.5 & 10533.75 \\
\hline Sids 40 "Station" & 3162.5 & 1581.25 & 3215.5 & 1607.75 & 2250 & 435 & 900 & 2250 & 675 & 750 & 1666 & 12088.5 & 10507.25 & 12141.5 & 10533.75 \\
\hline Clone 5 & 3162.5 & 1581.25 & 3215.5 & 1607.75 & 1125 & 435 & 900 & 2250 & 675 & 750 & 1666 & 10963.5 & 9382.25 & 11016.5 & 9408.75 \\
\hline Clone 10 & 3162.5 & 1581.25 & 3215.5 & 1607.75 & 1125 & 435 & 900 & 2250 & 675 & 75 & 1666 & 10963.5 & 9382.25 & 11016.5 & 9408.75 \\
\hline Clone 18 & 3162.5 & 1581.25 & 3215.5 & 1607.75 & 1125 & 435 & 900 & 2250 & 675 & 750 & 1666 & 10963.5 & 9382.25 & 11016.5 & 9408.75 \\
\hline Clone 22 W & 3162.5 & & 3215.5 & & 1125 & 435 & 900 & 2250 & 675 & 750 & 1666 & & 2.25 & 11016.5 & 9408.75 \\
\hline Clone 24 & 3162.5 & 1581.25 & 3215.5 & 1607.75 & 1125 & 435 & 900 & 2250 & 675 & 750 & 1666 & 10963.5 & 9382.25 & 11016.5 & 9408.75 \\
\hline Clone 25 & 3162.5 & 1581.25 & 3215.5 & 1607.75 & 1125 & 435 & 900 & 2250 & 675 & 750 & 1666 & 10963.5 & 9382.25 & 11016.5 & 9408.75 \\
\hline Egaseed clone & 3162.5 & 1581.25 & 3215.5 & 1607.75 & 1125 & 435 & 900 & 2250 & 675 & 750 & 1666 & 10963.5 & 9382.25 & 11016.5 & 9408.75 \\
\hline Egyptian & 3162.5 & 1581.25 & 3215.5 & 1607.75 & 1125 & 435 & 900 & 2250 & 675 & 750 & 1666 & 10963.5 & 9382.25 & 11016.5 & 9408.75 \\
\hline El-Wady & 3162.5 & 1581.25 & 3215.5 & 1607.75 & 1125 & 435 & 900 & 2250 & 675 & 750 & 1666 & 10963.5 & 9382.25 & 11016.5 & 9408.75 \\
\hline Owainat & 3162.5 & 1581.25 & 3215.5 & 1607.75 & 1125 & 435 & 900 & 2250 & 675 & 750 & 1666 & 10963.5 & 9382.25 & 11016.5 & 9408.75 \\
\hline Salaqus-3 W & 3162.5 & 1581.25 & 3215.5 & 1607.75 & 1125 & 435 & 900 & 2250 & 675 & 750 & 1666 & 10963.5 & 9382.25 & 11016.5 & 9408.75 \\
\hline
\end{tabular}

All numbers are in Egyptian Pounds

$\mathbf{1}_{\mathbf{1}}$ Seed cost estimated based on average seed price 4.5 L.E per $\mathrm{Kg}$ for coloured genotypes and 3.5 L.E for white genotypes 
Table 4. Economic feasibility for fresh yield of all garlic genotypes fertilized with different compost treatments

\begin{tabular}{|c|c|c|c|c|c|c|c|c|c|c|c|c|c|c|c|c|c|}
\hline \multirow[b]{3}{*}{ Genotypes } & \multicolumn{4}{|c|}{ Total fresh yield Ton/Fed. } & \multicolumn{4}{|c|}{ Gross as organic (L.E/Fed. $)^{1}$} & \multicolumn{4}{|c|}{ Net return $(\mathrm{NR})^{2}$, L.E } & \multirow{3}{*}{ 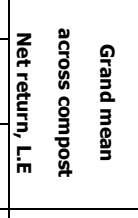 } & \multicolumn{4}{|c|}{ Profitability, \% (NR/TC)*100 } \\
\hline & \multicolumn{2}{|c|}{ Plant compost } & \multicolumn{2}{|c|}{ Plant-animal compost } & \multicolumn{2}{|c|}{ Plant compost } & \multicolumn{2}{|c|}{ Plant-animal compost } & \multicolumn{2}{|c|}{ Plant compost } & \multicolumn{2}{|c|}{ Plant-animal compost } & & Plant & ipost & $\begin{array}{r}\text { Plan } \\
\text { col }\end{array}$ & $\begin{array}{l}\text { mal } \\
\text { st }\end{array}$ \\
\hline & $\begin{array}{l}120 \mathrm{~kg} \\
\mathrm{~N} / \mathrm{Fed}\end{array}$ & $\begin{array}{l}60 \mathrm{~kg} \\
\mathrm{~N} / \mathrm{Fed}\end{array}$ & $\begin{array}{c}120 \mathrm{~kg} \\
\mathrm{~N} / \mathrm{Fed}\end{array}$ & $\begin{array}{l}60 \mathrm{~kg} \\
\mathrm{~N} / \mathrm{Fed}\end{array}$ & $\begin{array}{l}120 \mathrm{~kg} \\
\mathrm{~N} / \mathrm{Fed}\end{array}$ & $\begin{array}{l}60 \mathrm{~kg} \\
\mathrm{~N} / \mathrm{Fed}\end{array}$ & $\begin{array}{l}120 \mathrm{~kg} \\
\mathrm{~N} / \mathrm{Fed}\end{array}$ & $\begin{array}{l}60 \mathrm{~kg} \\
\mathrm{~N} / \mathrm{Fed}\end{array}$ & $\begin{array}{l}120 \mathrm{~kg} \\
\mathrm{~N} / \mathrm{Fed}\end{array}$ & $60 \mathrm{~kg} \mathrm{~N} / \mathrm{Fed}$ & $\begin{array}{l}120 \mathrm{~kg} \\
\mathrm{~N} / \text { Fed }\end{array}$ & $\begin{array}{l}60 \mathrm{~kg} \\
\mathrm{~N} / \mathrm{Fed}\end{array}$ & & $\begin{array}{c}120 \mathrm{~kg} \\
\mathrm{~N} / \mathrm{Fed}\end{array}$ & $\begin{array}{c}60 \mathrm{~kg} \\
\mathrm{~N} / \mathrm{Fed}\end{array}$ & $\begin{array}{l}120 \mathrm{~kg} \\
\mathrm{~N} / \mathrm{Fed}\end{array}$ & $\begin{array}{l}60 \mathrm{~kg} \\
\mathrm{~N} / \mathrm{Fed}\end{array}$ \\
\hline Aiat" clone 1 . & 3.004 & 2.018 & 1.901 & 0.9803 & 13518 & 9081 & 8554.5 & 4411.35 & 1429.5 & -1426.25 & -3587 & -6122.4 & -2426.54 & 11.83 & -13.57 & -29.54 & -58.12 \\
\hline Bani Ghany & 3.126 & 2.592 & 3.273 & 1.737 & 14067 & 11664 & 14728.5 & 7816.5 & 1978.5 & 1156.75 & 2587 & -2717.25 & 751.25 & 16.37 & 11.01 & 21.31 & -25.80 \\
\hline Clone 21 & 3.983 & 2.105 & 3.854 & 2.559 & 17923.5 & 9472.5 & 17343 & 11515.5 & 5835 & -1034.75 & 5201.5 & 981.75 & 2745.88 & 48.27 & -9.85 & 42.84 & 9.32 \\
\hline Clone $22 \mathrm{C}$ & 3.453 & 3.797 & 3.829 & 2.442 & 15538.5 & 17086.5 & 17230.5 & 10989 & 3450 & 6579.25 & 5089 & 455.25 & 3893.38 & 28.54 & 62.62 & 41.91 & 4.32 \\
\hline Egaseed 1 & 4.16 & 3.063 & 4.685 & 2.519 & 18720 & 13783.5 & 21082.5 & 11335.5 & 6631.5 & 3276.25 & 8941 & 801.75 & 4912.63 & 54.86 & 31.18 & 73.64 & 7.61 \\
\hline Egaseed 2 & 2.927 & 2.792 & 3.369 & 2.451 & 13171.5 & 12564 & 15160.5 & 11029.5 & 583 & 1556.75 & 2519 & -4.25 & 1163.63 & 4.63 & 14.14 & 19.93 & -0.04 \\
\hline Grower's Clone & 2.438 & 2.674 & 3.363 & 1.801 & 10971 & 12033 & 15133.5 & 8104.5 & -1117.5 & 1525.75 & 2992 & -2429.25 & 242.75 & -9.24 & 14.52 & 24.64 & -23.06 \\
\hline Salaqus-3 C & 3.719 & 2.682 & 3.328 & 2.515 & 16735.5 & 12069 & 14976 & 11317.5 & 4647 & 1561.75 & 2834.5 & 783.75 & 2456.75 & 38.44 & 14.86 & 23.35 & 7.44 \\
\hline Sids 40 "Aiat" & 2.714 & 2.51 & 4.081 & 2.474 & 12213 & 11295 & 18364.5 & 11133 & 124.5 & 787.75 & 6223 & 599.25 & 1933.63 & 1.03 & 7.50 & 51.25 & 5.69 \\
\hline Sids 40 "Station" & 3.135 & 3.236 & 2.566 & 1.512 & 14107.5 & 14562 & 11547 & 6804 & 2019 & 4054.75 & -594.5 & -3729.75 & 437.38 & 16.70 & 38.59 & -4.90 & -35.41 \\
\hline Clone 5 & 5.036 & 3.929 & 4.222 & 3.353 & 17626 & 13751.5 & 14777 & 11735.5 & 6662.5 & 4369.25 & 3760.5 & 2326.75 & 4279.75 & 60.77 & 46.57 & 34.14 & 24.73 \\
\hline Clone 10 & 2.188 & 2.637 & 3.217 & 1.777 & 7658 & 9229.5 & 11259.5 & 6219.5 & -3305.5 & -152.75 & 243 & -3189.25 & -1601.13 & -30.15 & -1.63 & 2.21 & -33.90 \\
\hline Clone 18 & 3.118 & 2.543 & 3.345 & 2.801 & 10913 & 8900.5 & 11707.5 & 9803.5 & -50.5 & -481.75 & 691 & 394.75 & 138.38 & -0.46 & -5.13 & 6.27 & 4.20 \\
\hline Clone 22 w & 2.678 & 3.288 & 3.486 & 2.197 & 9373 & 11508 & 12201 & 7689.5 & -1590.5 & 2125.75 & 1184.5 & -1719.25 & 0.13 & -14.51 & 22.66 & 10.75 & -18.27 \\
\hline Clone 24 & 3.448 & 3.513 & 3.896 & 3.252 & 12068 & 12295.5 & 13636 & 11382 & 1104.5 & 2913.25 & 2619.5 & 1973.25 & 2152.63 & 10.07 & 31.05 & 23.78 & 20.97 \\
\hline Clone 25 & 3.762 & 3.806 & 3.498 & 2.046 & 13167 & 13321 & 12243 & 7161 & 2203.5 & 3938.75 & 1226.5 & -2247.75 & 1280.25 & 20.10 & 41.98 & 11.13 & -23.89 \\
\hline Egaseed clone & 3.941 & 3.189 & 3.21 & 2.793 & 13793.5 & 11161.5 & 11235 & 9775.5 & 2830 & 1779.25 & 218.5 & 366.75 & 1298.63 & 25.81 & 18.96 & 1.98 & 3.90 \\
\hline Egyptian & 3.412 & 4.247 & 4.109 & 2.986 & 11942 & 14864.5 & 14381.5 & 10451 & 978.5 & 5482.25 & 3365 & 1042.25 & 2717.00 & 8.93 & 58.43 & 30.55 & 11.08 \\
\hline El-Wady & 4.27 & 4.459 & 4.897 & 4.357 & 14945 & 15606.5 & 17139.5 & 15249.5 & 3981.5 & 6224.25 & 6123 & 5840.75 & 5542.38 & 36.32 & 66.34 & 55.58 & 62.08 \\
\hline Owainat & 2.664 & 2.348 & 2.479 & 1.886 & 9324 & 8218 & 8676.5 & 6601 & -1639.5 & -1164.25 & -2340 & -2807.75 & -1987.88 & -14.95 & -12.41 & -21.24 & -29.84 \\
\hline Salaqus-3 $\mathbf{w}$ & 2.727 & 2.642 & 2.776 & 2.712 & 9544.5 & 9247 & 9716 & 9492 & -1419 & -135.25 & -1300.5 & 83.25 & -692.88 & -12.94 & -1.44 & -11.81 & 0.88 \\
\hline Average & 3.33 & 3.05 & 3.49 & 2.44 & 13205.69 & 11986.38 & 13861.57 & 9524.59 & 1682.67 & 2044.61 & 2285.55 & -443.69 & 1392.28 & 14.31 & 20.78 & 19.42 & -4.10 \\
\hline
\end{tabular}

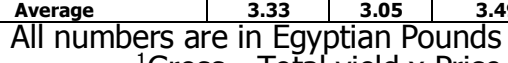

${ }^{1}$ Gross= Total yield $x$ Price as organic 4.5 L.E per Kg for coloured genotypes and 3.5 L.E for white genotypes

Net return $(N R)=$ Gross - Total cost 
Table 5. Economic feasibility for cured yield of all garlic genotypes fertilized with different compost treatments

\begin{tabular}{|c|c|c|c|c|c|c|c|c|c|c|c|c|c|c|c|c|c|}
\hline \multirow{3}{*}{ Genotypes } & \multicolumn{4}{|c|}{ Total cured yield Ton/Fed. } & \multicolumn{4}{|c|}{ 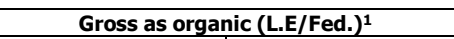 } & \multicolumn{4}{|c|}{ Net return $(\mathrm{NR})^{2}$, L.E } & \multirow{3}{*}{ 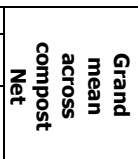 } & \multicolumn{4}{|c|}{ Profitability, \% (NR/TC)*100 } \\
\hline & \multicolumn{2}{|c|}{ Plant compost } & \multicolumn{2}{|c|}{$\begin{array}{c}\text { Plant-animal } \\
\text { compost }\end{array}$} & \multicolumn{2}{|c|}{ Plant compost } & \multicolumn{2}{|c|}{$\begin{array}{c}\text { Plant-animal } \\
\text { compost }\end{array}$} & \multicolumn{2}{|c|}{ Plant compost } & \multicolumn{2}{|c|}{$\begin{array}{c}\text { Plant-animal } \\
\text { compost }\end{array}$} & & \multicolumn{2}{|c|}{ Plant compost } & \multicolumn{2}{|c|}{$\begin{array}{c}\text { Plant-animal } \\
\text { compost }\end{array}$} \\
\hline & $\begin{array}{c}120 \\
\mathrm{~kg} \\
\mathrm{~N} / \mathrm{Fed}\end{array}$ & $\begin{array}{l}60 \mathrm{~kg} \\
\mathrm{~N} / \mathrm{Fed}\end{array}$ & $\begin{array}{l}120 \mathrm{~kg} \\
\mathrm{~N} / \mathrm{Fed}\end{array}$ & $\begin{array}{l}60 \mathrm{~kg} \\
\mathrm{~N} / \mathrm{Fed}\end{array}$ & $\begin{array}{l}120 \mathrm{~kg} \\
\mathrm{~N} / \mathrm{Fed}\end{array}$ & $\begin{array}{l}60 \mathrm{~kg} \\
\mathrm{~N} / \mathrm{Fed}\end{array}$ & $\begin{array}{l}120 \mathrm{~kg} \\
\mathrm{~N} / \mathrm{Fed}\end{array}$ & $\begin{array}{l}60 \mathrm{~kg} \\
\mathrm{~N} / \mathrm{Fed}\end{array}$ & $\begin{array}{l}120 \mathrm{~kg} \\
\mathrm{~N} / \mathrm{Fed}\end{array}$ & $\begin{array}{l}60 \mathrm{~kg} \\
\mathrm{~N} / \mathrm{Fed}\end{array}$ & $\begin{array}{l}120 \mathrm{~kg} \\
\mathrm{~N} / \mathrm{Fed}\end{array}$ & $\begin{array}{l}60 \mathrm{~kg} \\
\mathrm{~N} / \mathrm{Fed}\end{array}$ & & $\begin{array}{l}120 \mathrm{~kg} \\
\mathrm{~N} / \mathrm{Fed}\end{array}$ & $\begin{array}{l}60 \mathrm{~kg} \\
\mathrm{~N} / \mathrm{Fed}\end{array}$ & $\begin{array}{l}120 \mathrm{~kg} \\
\mathrm{~N} / \mathrm{Fed}\end{array}$ & $\begin{array}{l}60 \mathrm{~kg} \\
\mathrm{~N} / \mathrm{Fed}\end{array}$ \\
\hline Aiat" clone 1 " & 2.045 & 1.513 & 1.366 & 0.633 & 13497 & 9985.8 & 9015.6 & 4177.8 & 908.5 & -1021.45 & -3625.9 & -6855.95 & -2648.7 & 7.22 & -9.28 & -28.68 & 62.14 \\
\hline Bani Ghany & 2.332 & 1.959 & 2.452 & 1.253 & 15391.2 & 12929.4 & 16183.2 & 8269.8 & 2802.7 & 1922.15 & 3541.7 & -2763.95 & 1375.65 & 22.26 & 17.46 & 28.02 & 25.05 \\
\hline Clone 21 & 2.794 & 1.55 & 2.585 & 1.746 & 18440.4 & 10230 & 17061 & 11523.6 & 5851.9 & -777.25 & 4419.5 & 489.85 & 2496 & 46.49 & -7.06 & 34.96 & 4.44 \\
\hline Clone 22 C & & & & & 427.4 & & 18407.4 & 11147.4 & 3838.9 & & 5765.9 & & & 30.50 & 63.87 & 45.61 & 1.03 \\
\hline Egaseed 1 & 2.948 & 2.231 & 3.445 & 1.766 & 19456.8 & 14724.6 & 22737 & 11655.6 & 6868.3 & 3717.35 & 10095.5 & 621.85 & 5325.75 & 54.56 & 33.77 & 79.86 & 5.64 \\
\hline Egaseed 2 & 1.881 & 1.851 & 2.198 & 1.496 & 12414.6 & 12216.6 & 14506.8 & 9873.6 & -673.9 & 709.35 & 1365.3 & -1660.15 & -64.85 & -5.15 & 6.16 & 10.39 & \\
\hline Grower's Clone & 1.69 & 2.007 & 2.487 & 1.249 & 11154 & 13246.2 & 16414.2 & 8243.4 & -1434.5 & 2238.95 & 3772.7 & -2790.35 & 446.7 & -11.40 & 20.34 & 29.84 & 25,29 \\
\hline Salaqus-3 & 2.783 & & 25 & & 1836 & & 16500 & & & & 3858.5 & & & & & 52 & 8.69 \\
\hline Sids 40 "Aiat" & 2.11 & 1.859 & 2.655 & 1.727 & 13926 & 12269.4 & 17523 & 11398.2 & 1337.5 & 1262.15 & 4881.5 & 364.45 & 1961.4 & 10.62 & 11.47 & 38.61 & 3.30 \\
\hline Sids 40 "Station" & 2.361 & 2.334 & 1.909 & 1.067 & 15582.6 & 15404.4 & 12599.4 & 7042.2 & 2994.1 & 4397.15 & -42.1 & -3991.55 & 839.4 & 23.78 & 39.95 & -0.33 & 36.18 \\
\hline Clone 5 & 3.067 & 2.481 & 2.65 & 2.45 & 17175.2 & 13893.6 & 14840 & 13720 & 5711.7 & 4011.35 & 3323.5 & 3811.25 & 4214.45 & 49.83 & 40.59 & 28.86 & 38.46 \\
\hline Clone 10 & 1.322 & 1.652 & 2.007 & 1.133 & 7403.2 & 9251.2 & 11239.2 & 6344.8 & -4060.3 & -631.05 & -277.3 & -3563.95 & -2133.15 & -35.42 & -6.39 & -2.41 & \\
\hline Clone 18 & 1.883 & 1.654 & 2.1 & 1.77 & 10544.8 & 9262.4 & 11760 & 9912 & -918.7 & -619.85 & 243.5 & 3.25 & -322.95 & -8.01 & -6.27 & 2.11 & 0.03 \\
\hline Clone 22 w & 1.627 & 1.95 & 2.088 & 1.338 & 9111.2 & 10920 & 11692.8 & 7492.8 & -2352.3 & 1037.75 & 176.3 & -2415.95 & -888.55 & -20.52 & 10.50 & 1.53 & 24.38 \\
\hline Clone 24 & 2.029 & 2.029 & 2.4 & 1.871 & 11362.4 & 11362.4 & 13440 & 10477.6 & -101.1 & 1480.15 & 1923.5 & 568.85 & 967.85 & -0.88 & 14.98 & 16.70 & 5.74 \\
\hline Clone 25 & 2.288 & 2.465 & 2.128 & 1.285 & 12812.8 & 13804 & 11916.8 & 7196 & 1349.3 & 3921.75 & 400.3 & -2712.75 & 739.65 & 11.77 & 39.68 & 3.48 & 27.38 \\
\hline Egaseed clone & 2.072 & 2.053 & 1.981 & 1.131 & 11603.2 & 11496.8 & 11093.6 & 6333.6 & 139.7 & 1614.55 & -422.9 & -3575.15 & -560.95 & 1.22 & 16.34 & -3.67 & 36.08 \\
\hline & & & & & & & 13865.6 & & & & 2349.1 & & & & & 40 & 1.90 \\
\hline El-Wady & 2.51 & 2.792 & 3.029 & 2.661 & & 15635.2 & 16962.4 & 14901.6 & 2592.5 & 5752.95 & 5445.9 & 4992.85 & & 22.62 & 58.21 & 47.29 & 50.39 \\
\hline Owainat & 1.588 & 1.442 & 1.52 & 1.166 & 8892.8 & 8075.2 & 8512 & 6529.6 & -2570.7 & -1807.05 & -3004.5 & -3379.15 & -2690.35 & -22.43 & 18.29 & -26.09 & 34.10 \\
\hline Salaqus-3W & 1.531 & 1.587 & 1.7 & 1.646 & 8573.6 & 8887.2 & 9520 & 9217.6 & -2889.9 & -995.05 & -1996.5 & -691.15 & -1643.15 & -25.21 & 10.07 & -17.34 & -6.98 \\
\hline Average & 2.16 & 2.03 & 2.31 & 1.56 & $\begin{array}{c}13211 . \\
32\end{array}$ & $\begin{array}{c}12349 . \\
73\end{array}$ & $\begin{array}{c}14085 . \\
24\end{array}$ & $\begin{array}{c}9406.9 \\
6\end{array}$ & $\begin{array}{c}1188.3 \\
0\end{array}$ & 1907.96 & $\begin{array}{c}2009.2 \\
1\end{array}$ & 1061.31 & 1011.04 & 9.33 & $\begin{array}{c}18.2 \\
3\end{array}$ & 16.17 & -9.92 \\
\hline
\end{tabular}

All numbers are in Egyptian Pounds

${ }^{1}$ Gross $=$ Total yield $x$ Price as organic 6.6 L.E per $\mathrm{Kg}$ for coloured genotypes and $5.6 \mathrm{~L} . \mathrm{E}$ for white genotypes

2 Net return $(N R)=$ Gross - Total variable cost as mentioned in Table $1+500$ L.E for storage process 


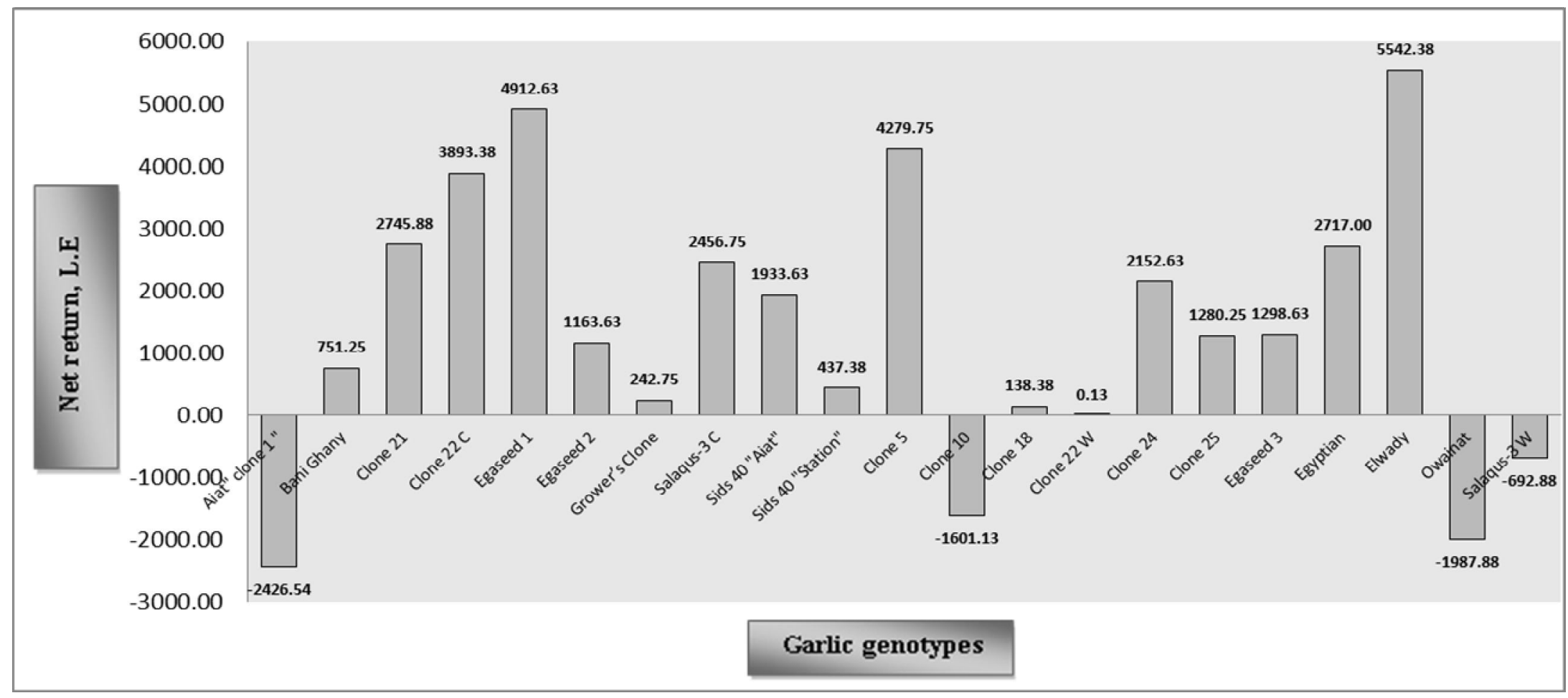

Fig. 1. Average net return of fresh garlic genotypes across the tested treatments. 


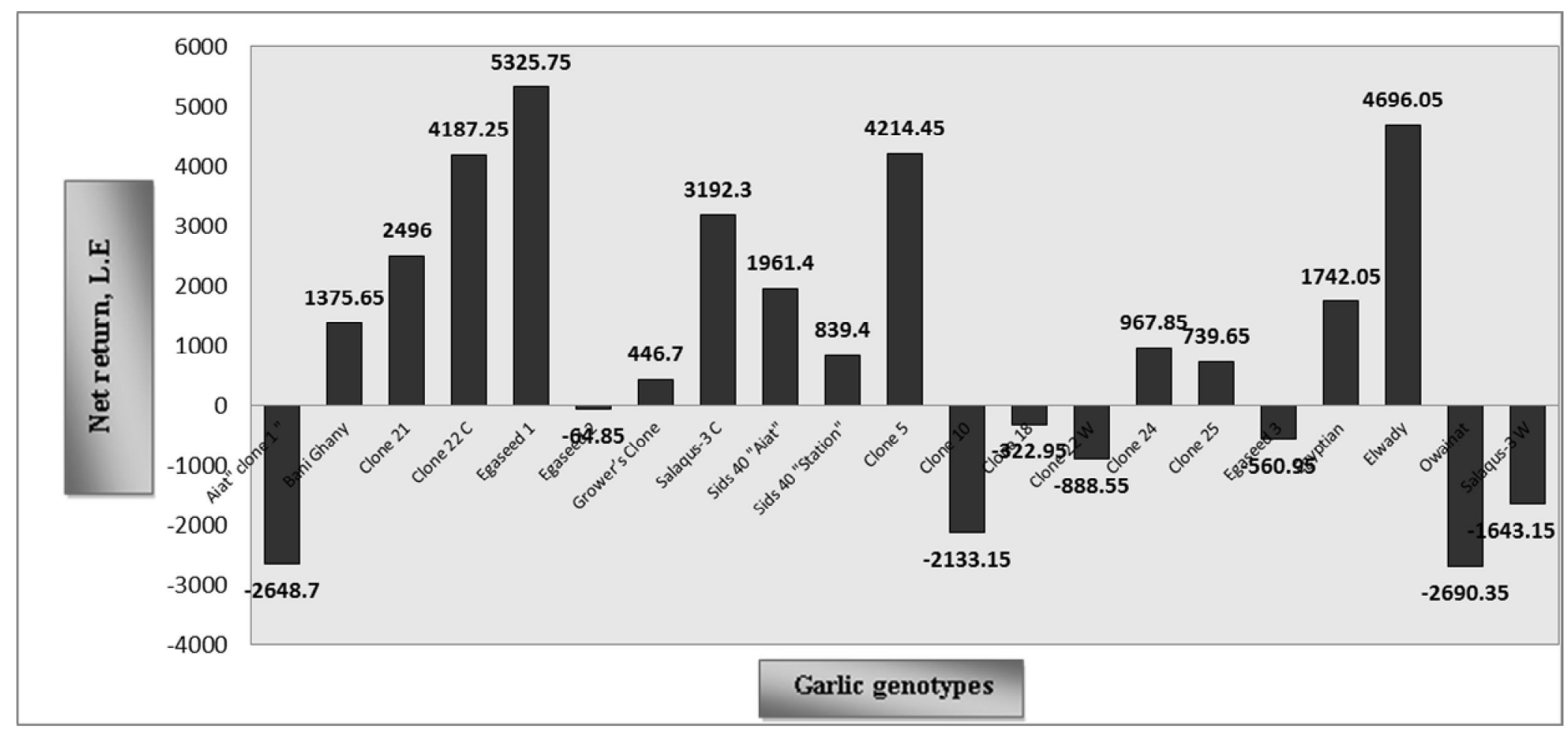

Fig. 2. Average net return of cured garlic genotypes across the tested treatments. 


\section{REFERENCES}

1. Abd El Kareem, S. M. A. 2015. Effect of organic and bio-fertilization on yield and quality of onion crop.. Ph. D. thesis, Faculty of Agric. Sohag.Univ. 103 pp.

2. Besheit, S. Y.; Abo El Wafa, A. M.; Abo El Hamed, A. S. and Bekheet, M. A. 2002. Quality and productivity of sugar beet as affected by intercropping onion in various densities. Al Azher J. Agric. Res., 36:87-101.

3. Conner, D. and Rangarajan, A. 2009. Production costs of organic vegetable farms: two case studies from Pennsylvania. Hort. Technology, 19(1):193-199.

4. Dahshan, A. M. A. 2013. Influence of phosphorus as mineral and bio-fertilizers on growth and yield of potatoes.M. Sc. thesis, Faculty of Agric. El-Minia. Univ. 149 pp.

5. Estes, E.A.;Kleese, T. and Lauffer, L. 2003. North Carolina organic vegetable production cost study. ARE Res. Rpt. No. 31, Department of Agricultural and Resource Economics, North Carolina State Univ., Raleigh.

6. Frank, G. G. 1997. Enterprise accounting.1-9.

7. Haque, M. A.; Monayem-Miah, M. A.; Hossain, M. S.; Luna, A. N. and Rahman, K. S. 2013. Profitability of garlic (Allium sativum L.) cultivation in some selected areas of Bangladesh. Bangladesh J. Agril. Res. 38(4):589-598.

8. Hasan, M. K.; Islam, M. S. and Mahmud, M. A. A. 2012. Present status profitability and potentiality of garlic production in Bangladesh. International Journal of Sustainable Agricultural Technology, 8(7):1-7.

9. Meena, L. K.; Sen, C. and Bairwa, S. 2013. Economics of garlic production in baran district of rajasthan; break even analysis. Asian Journal of Agriculture and Rural Development, 3(10):697-701.

10. O'Dell, C .R. (1984). Selected production cost budgets for 29 horticultural crops. Virginia Cooperative Extension Service. 60pp.

11. Post, E. and Schahczenski, J. (eds). 2012. Understanding organic pricing and costs of production. Report: National Sustainable Agriculture Information Service 12pp.

12. Richards, T. J. (2011). The economics of the organic food system: discussion. American journal of agriculturalEconomics 94(2):322-323.

13. Sanusi, M. M. and Ayinde, I. A. 2013. Profitability of pepper production in derived savannah zone of Ogun State, Nigeria. IJAFS 4(1\&2):401-410.

14. The University of California at Davis. 2008. Cost and return studies.

15. Toaima, S. E. A.; El Douby, K. A. and Naffi, A. I. 2001. Effect of different intercropping system of onion chemical analysis. Egypt J. Agric. Res. 79(3):9831004. 
تقدير التكاليف والعائد للثوم العضوي تحت ظروف الأراضي المزروعة حديثاً ، بالئا

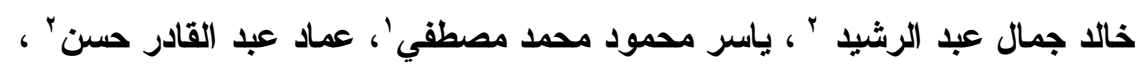

$$
\begin{aligned}
& \text { يوسف يوسف عبد العاطي' سيف النصر حسين جاد الحق' } \\
& \text { ا- - قسم البساتين - كلية الزراعة - جامعة المنيا - المنيا - جمهورية مصر العربية. }
\end{aligned}
$$

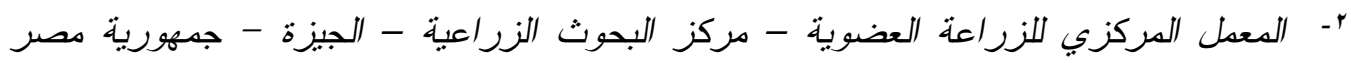

$$
\begin{aligned}
& \text { العربية. }
\end{aligned}
$$

في دراسة إقتصادية أولية لإنتاج الثوم العضوي في الأر اضي الجديدة تحت ظروف محافظة

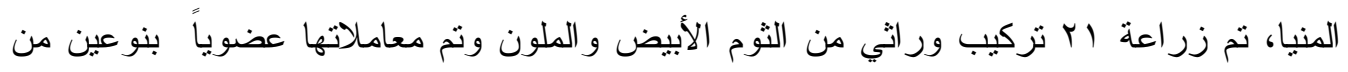

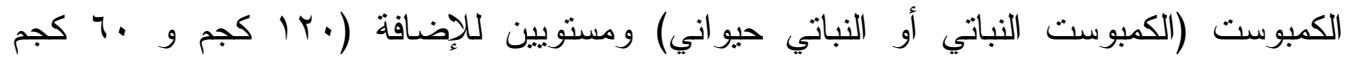

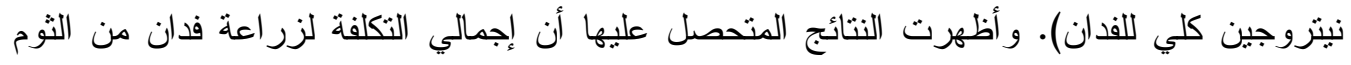

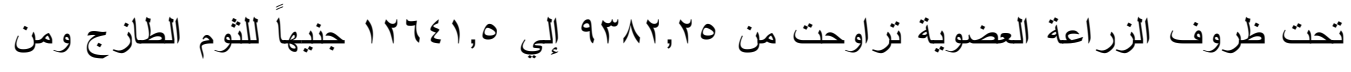

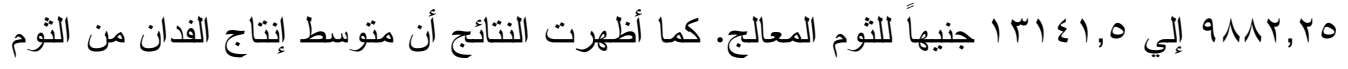

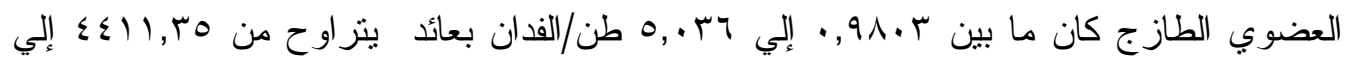

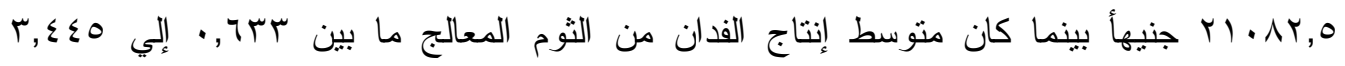

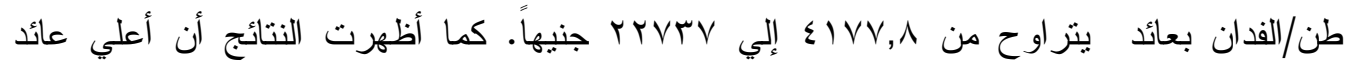

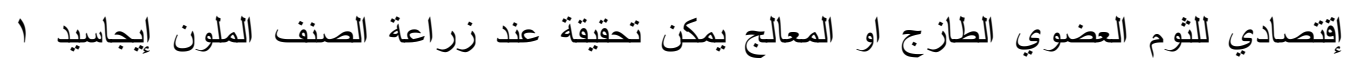

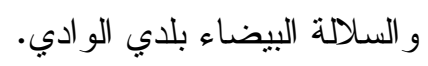

